\title{
On Some Topological Properties of Pessimistic Multigranular Rough Sets
}

\author{
B.K.Tripathy \\ SCSE, VIT University, Vellore-632 014, Tamil Nadu, INDIA \\ tripathybk@vit.ac.in \\ M. Nagaraju \\ SCSE, VIT University, Vellore-632 014, Tamil Nadu, INDIA \\ mnagaraju@vit.ac.in
}

\begin{abstract}
Rough set theory was introduced by Pawlak as a model to capture impreciseness in data and since then it has been established to be a very efficient tool for this purpose. The definition of basic rough sets depends upon a single equivalence relation defined on the universe or several equivalence relations taken one each at a time. There have been several extensions to the basic rough sets introduced since then in the literature. From the granular computing point of view, research in classical rough set theory is done by taking a single granulation. It has been extended to multigranular rough set (MGRS) model, where the set approximations are defined by taking multiple equivalence relations on the universe simultaneously. Multigranular rough sets are of two types; namely optimistic MGRS and pessimistic MGRS. Topological properties of rough sets introduced by Pawlak in terms of their types were studied by Tripathy and Mitra to find the types of the union, intersection and complement of such sets. Tripathy and Raghavan have extended the topological properties of basic single granular rough sets to the optimistic MGRS context. Incomplete information systems take care of missing values for items in data tables. MGRS has also been extended to such type of incomplete information systems. In this paper we have carried out the study of topological properties of pessimistic MGRS by finding out the types of the union, intersection and complement of such sets. Also, we have provided proofs and examples to illustrate that the multiple entries in the table can actually occur in practice. Our results hold for both complete and incomplete information systems. The multiple entries in the tables occur due to imp reciseness and ambiguity in the information. This is very common in many of the real life situations and needed to be addressed to handle such situations in efficient manner.
\end{abstract}

Index Terms - Rough Sets, Equivalence Relations, Tolerance Relations, Type of Rough Sets, Multi Granular Rough Sets

\section{Introduction}

The main observations of our traditional tools for formal modeling, reasoning and computing are crisp, deterministic and preciseness nature, which restrict their applicability in real life situations, led to the extension of the concept of crisp sets so as to model imprecise data and enhance their modeling power. One such approach to capture imprec iseness was due to Pawlak [5, 6], who introduced the notion of Rough Sets, which is an excellent tool to capture impreciseness in data. The basic assumption of rough set theory is that human knowledge about a universe depends upon their capability to classify its objects. Classifications of a universe and equivalence relations defined on it are known to be interchangeable notions. So, for mathematical reasons equivalence relations were considered by Pawlak to define rough sets. A rough set is represented by a pair of crisp sets, called the lower approximation comprises of elements, which belong to it definitely and upper approximation comprises of elements, which are possibly in the set with respect to the available information.

To improve the modeling capability of basic rough sets several extensions have been made in different directions. One such extension is the rough sets based upon tolerance relations instead of equivalence relations. These rough sets are sometimes called incomplete rough set models. In the view of granular computing, classical rough set theory is researched by a single granulation. The basic rough set model has been extended to rough set model based on multigranulations (MGRS) in [10], where the set approximations are defined by using mu lti-equivalences on the universe. Using similar concepts, that is taking multiple tolerance relations instead of multiple equivalence relations; incomplete rough set model based on multi-granulations was introduced in [11]. Several fundamental properties of these types of rough sets have been studied [10,11, 12].

Employing the notions of lower and upper approximations of rough sets, an interesting 
characterization of rough sets has been made by Pawlak in [6], where he introduced the types (originally called kinds) of rough sets. There are two different ways of characterizing rough sets; the accuracy coefficient and the topological characterization introduced through the notion of types. As mentioned by Pawlak himself [6], in practical applications of rough sets we combine both types of information about the borderline region, that is, of the accuracy of measure as well as the information about the topological classification of the set under consideration. Keeping this in mind, Tripathy and Mitra [16] have studied the types of rough sets by finding out the types of union and intersection of rough sets of different types. These results were extended to the context of optimistic multi granular rough sets by Tripathy et al [17]. In this paper, we study these results for the pessimistic multi granular context, which also remains the same for both the complete and incomplete cases.

\section{Definitions And Notations}

Let $U$ be a universe of discourse and $R$ be an equivalence relation over $U$. By $U / R$ we denote the family of all equivalence classes of $R$, referred to as categories or concepts of $R$ and the equivalence class of an element $x \in U$ is denoted by $[\mathrm{x}]_{R}$. By a knowledge base, we understand a relational system $K=(U, P)$, where $U$ is as above and $P$ is a family of equivalence relations over $U$. For any subset $\mathrm{Q}(\neq \phi) \subseteq \mathrm{P}$, the intersection of all equivalence relations in $\mathrm{Q}$ is denoted by $\operatorname{IND}(\mathrm{Q})$ and is called the indiscernibility relation over Q. Given any $X \subseteq U$ and $\mathrm{R} \in \mathrm{IND}(\mathrm{K})$, we associate two subsets, $\underline{R} X=\bigcup\{Y \in U / R: Y \subseteq X\}$ and $\bar{R} X=\bigcup\{Y \in U / R: Y \cap X \neq \phi\}$, called the R-lower and Rupper approximations of $X$ respectively. The $\mathrm{R}$ boundary of $X$ is denoted by $B N_{R}(X)$ and is given by $B N_{R}(X)=\bar{R} X-\underline{R} X$. The elements of $\underline{R} \mathrm{X}$ are those elements of $U$, which can certainly be classified as elements of $\mathrm{X}$, and the elements of $\bar{R} \mathrm{X}$ are those elements of $U$, which can possibly be classified as elements of $\mathrm{X}$, employing knowledge of $R$. We say that $\mathrm{X}$ is rough with respect to $\mathrm{R}$ if and only if $\underline{R} X \neq \bar{R} X$, equivalently $B N_{R}(X) \neq \phi . \quad \mathrm{X}$ is said to be $\mathrm{R}$ definable if and only if $\underline{R} X=\bar{R} X$, or $B N_{R}(X)=\phi$.

In the view of granular computing (proposed by L. A. Zadeh), an equivalence relation on the universe can be regarded as a granulation, and a partition on the universe can be regarded as a granulation space [2, 3]. For an incomplete information system, similarly, a tolerance relation on the universe can be regard as a granulation, and a cover induced by the relation can be regarded as a granulation space. Several measures in knowledge base closely associated with granular computing, such as knowledge granulation, granulation measure, in formation entropy and rough entropy, were discussed in [2, 3, 4]. On research of rough set method based on multi-g ranulations, Y. H. Qian and J. Y. Liang brought forward a rough set model based on multigranulations [10], which is established by using multi equivalence relations. In [11] an extension of MGRS, rough set model based on multi tolerance relations in incomplete information systems is developed.

We define below the optimistic MGRS.

Definition 2.1: Let $K=(U, \mathbf{R})$ be a knowledge base, $\mathbf{R}$ be a family of equivalence relations, $\mathrm{X} \subseteq \mathrm{U}$ and $\mathrm{P}, \mathrm{Q} \in \mathbf{R}$. We define the optimistic multi-granular lower approximation and upper approximation of $\mathrm{X}$ in $\mathrm{U}$ as

$$
\underline{P+Q X}=\bigcup\left\{x /[x]_{P} \subseteq X \text { or }[x]_{Q} \subseteq X\right\}
$$

and

$$
\left.\overline{P+Q} X=\underline{(P+Q}\left(X^{C}\right)\right)^{C}
$$

Another kind of multi-granular rough sets called pessimistic multi-granular rough sets was introduced by Quian et al [13]. Now, they call the above type of multigranular rough sets as the optimistic multi-granular rough sets (which was introduced as the multi-granular rough sets [10]).

We define below the pessimistic multi-granular rough sets (PMGRS).

Definition 2.2: Let $K=(U, \mathbf{R})$ be a knowledge base, $\mathbf{R}$ be a family of equivalence relations, $\mathrm{X} \subseteq \mathrm{U}$ and $\mathrm{P}, \mathrm{Q} \in \mathbf{R}$. We define the pessimistic multi-granular lower approximation and upperapproximation of $\mathrm{X}$ in $\mathrm{U}$ as

$$
(\underline{P+Q})^{P} X=\bigcup\{x /[x] \subseteq X \text { and }[x] \subseteq X
$$

and

$$
\left.(\overline{P+Q})^{P} X=(\underline{(P+Q)})^{P}\left(X^{C}\right)\right)^{C}
$$

We state below several properties of pessimistic multi-granular rough sets (PMGRS) from [13].

Property 2.1: Let $K=(U, \mathbf{R})$ be a knowledge base, $\mathbf{R}$ be a family of equivalence re lations, $\mathrm{X} \subseteq \mathrm{U}$ and $\mathrm{P}, \mathrm{Q} \in \mathbf{R}$. The following properties hold true.

$$
\begin{aligned}
& \text { (2.4) } \frac{(P+Q)}{P}^{P}(X) \subseteq X \subseteq \overline{(P+Q)}^{P}(X) \\
& \text { (2.5) } \underline{(P+Q)}^{P}(\phi)=\phi=\overline{(P+Q)}^{P}(\phi),\left(\underline{(P+Q)}{ }^{P}(U)=U=\overline{(P+Q)}^{P}(U)\right. \\
& \text { (2.6) } \left.\frac{(P+Q)}{P}^{P}(X C)=\overline{((P+Q)}^{P}(X)\right)^{C} \\
& \text { (2.7) } \frac{(P+Q)}{(P)}^{P}(X)=\underline{P} X \cap \underline{Q} X \\
& \text { (2.8) } \overline{(P+Q)}^{P}(X)=\bar{P} X \cup \bar{Q} X \\
& \text { (2.9) } \underline{(P+Q)}^{P}(X)=\underline{(Q+P)}^{P}(X), \overline{(P+Q)}^{P}(X)=\overline{(Q+P)}^{P}(X)
\end{aligned}
$$


Property 2.2: Let $\mathrm{K}=(\mathrm{U}, \mathbf{R})$ be a knowledge base, $\mathbf{R}$ be a family of equivalence relations, $\mathrm{X}, \mathrm{Y} \subseteq \mathrm{U}$ and $\mathrm{P}, \mathrm{Q} \in \mathbf{R}$. The following properties hold true.

$$
\begin{aligned}
& \text { (2.10) } \frac{(P+Q)}{{ }^{P}}(X \cap Y)=\underline{(P+Q)}^{P} X \cap \underline{(P+Q)}^{P} Y \\
& \text { (2.11) }
\end{aligned}
$$

Next, we define PMGRS in incomplete information systems.

Definition 2.3: An information systemis a pair $\mathrm{S}=(\mathrm{U}, \mathrm{A})$, where $\mathrm{U}$ is a non-empty finite set of objects, A is a non-empty finite set of attributes. For every $a \in A$, there is a mapping $a: U \rightarrow V_{a}$, where $V_{a}$ is called the value set of a.

If $V_{a}$ contains a null value for at least one attribute $a \in A$, then $\mathrm{S}$ is called an incomplete information system. Otherwise, it is complete.

Definition 2.4: Let $\mathrm{S}=(U, A)$ be an incomplete information system, $\mathrm{P} \subseteq \mathrm{A}$ an attribute set. We define a binary relation on $\mathrm{U}$ as follows

$$
\begin{array}{r}
\operatorname{SIM}(\mathrm{P})=\{(\mathrm{u}, \mathrm{v}) \in \mathrm{UX} \mathrm{U} \mid \forall \mathrm{a} \in \mathrm{P}, \\
\mathrm{a}(\mathrm{u})=\mathrm{a}(\mathrm{v}) \text { or } \mathrm{a}(\mathrm{u})=* \text { or } \mathrm{a}(\mathrm{v})=*\} .
\end{array}
$$

In fact, $\operatorname{SIM}(P)$ is a tolerance relation on $U$, the concept of a tolerance relation has a wide variety of applications in classifications [1, 8].

$$
\text { It can be shown that } \operatorname{SIM}(\mathrm{P})=\bigcap_{a \in P} \operatorname{SIM}(\{\mathrm{A}\}) .
$$

Let $\mathrm{S}_{p}(\mathrm{u})$ denote the set $\{\mathrm{v} \in U \mid(\mathrm{u}, \mathrm{v}) \in \operatorname{SIM}(\mathrm{P})\}$. $\mathrm{S}_{P}(\mathrm{u})$ is the maximal set of objects which are possibly indistinguishable by $\mathrm{P}$ with $\mathrm{u}$.

Let $\mathrm{U} / \mathrm{SIM}(\mathrm{P})$ denote the family sets $\left\{\mathrm{S}_{p}(\mathrm{u}) \mid \mathrm{u} \in \mathrm{U}\right\}$, the classification or the knowledge induced by $\mathrm{P}$. A member $\mathrm{S}_{p}(\mathrm{u})$ from $\mathrm{U} / \mathrm{SIM}(\mathrm{P})$ will be called a tolerance class or an information granule. It should be noticed that the tolerance classes in $\operatorname{U} / \operatorname{SIM}(\mathrm{P})$ do not constitute a partition of $U$ in general. They constitute a cover of $\mathrm{U}$, i.e., $\mathrm{S}_{p}(\mathrm{u}) \neq \phi$ for every $\mathrm{u} \in \mathrm{U}$, and $\bigcup_{u \in U} \mathrm{~S}_{\mathrm{p}}(\mathrm{u})=\mathrm{U}$.

Definition 2.5: Let $\mathrm{S}=(\mathrm{U}, \mathrm{A})$ be an incomplete information system, $\mathrm{P}, \mathrm{Q} \subseteq \mathrm{A}$ two attribute subsets, $\mathrm{X}$ $\subseteq \mathrm{U}$, we define a lower approximation of $\mathrm{x}$ and a upper approximation of $\mathrm{x}$ in $\mathrm{U}$ by the following
(2.15) $\underline{(P+Q)}^{P} \mathrm{X}=\bigcup\left\{\mathrm{x} \mid \mathrm{SIM}_{P}(\mathrm{x}) \subseteq \mathrm{X}\right.$ and $\left.\operatorname{SIM}_{Q}(\mathrm{x}) \subseteq \mathrm{X}\right\}$

and

$$
\overline{(P+Q)}^{P}(\mathrm{X})=\left(\underline{(P+Q)}^{P}\left(\mathrm{X}^{c}\right)\right)^{c}
$$

A Multi-granulation Rough Set can be classified into following four types:

Definition 2.6: Let $K=(U, \mathbf{R})$ be a knowledge base, $\mathbf{R}$ be a family of equivalence re lations, $\mathrm{X} \subseteq \mathrm{U}$ and $\mathrm{P}, \mathrm{Q} \in \mathbf{R}$. Then

(2.16) If $\underline{(P+Q)}^{P}(\mathrm{X}) \neq \phi$ and $\overline{(P+Q)}^{P} \neq \mathrm{U}$, then we say that $\mathrm{X}$ is pessimistic roughly $\mathrm{P}+\mathrm{Q}$-definable.

(2.17) If $\underline{(P+Q)}^{P}(\mathrm{X})=\phi$ and $\overline{(P+Q)}^{P} \neq \mathrm{U}$, then we say that $\mathrm{X}$ is pessimistic internally $\mathrm{P}+\mathrm{Q}$-undefinable.

(2.18). If $\underline{(P+Q)}^{P}(\mathrm{X}) \neq \phi$ and $\overline{(P+Q)}^{P}=\mathrm{U}$, then we say that $\mathrm{X}$ is pessimistic externally $\mathrm{P}+\mathrm{Q}$ undefinable.

(2.19) If $\underline{(P+Q)}^{P}(\mathrm{X})=\phi$ and $\overline{(P+Q)}^{P}=\mathrm{U}$, then we say that $\mathrm{X}$ is pessimistic totally $\mathrm{P}+\mathrm{Q}-$ undefinable.

\section{Results}

In this section we shall find out the types of pessimistic multi granular rough sets (PMGRS). There are four sets of results accumulated in four tables. The first provides the type of a $\mathrm{P}+\mathrm{Q}$ rough set from the types of its $\mathrm{P}$ and $\mathrm{Q}$ rough set types. The second table provides the types of the complement of a multi granular rough set. In the third table we obtain the types for the union of two multi granular rough sets of all possible types. Similarly we establish the types of the intersection of two multi granular rough sets of all possible types. These results will be useful for further studies in approximation of classifications and rule generation.

\subsection{Table for type of $\mathrm{X}$ with respect to $(P+Q)^{P}$}

This subsection provides the type of a $P+Q$ rough set from the types of its $\mathrm{P}$ and $\mathrm{Q}$ rough set types in the following table. Proofs with examples for some of the entries in the table are then given.

\begin{tabular}{|c|c|c|c|c|c|}
\hline & \multicolumn{5}{|c|}{ Type of X with respect to Q } \\
\hline \multirow{4}{*}{$\begin{array}{c}\text { Type } \\
\text { of X } \\
\text { with } \\
\text { respect } \\
\text { to P }\end{array}$} & & $\mathrm{T}-1$ & $\mathrm{~T}-2$ & $\mathrm{~T}-3$ & $\mathrm{~T}-4$ \\
\cline { 2 - 6 } & $\mathrm{T}-1$ & $\mathrm{~T}-1$ & $\mathrm{~T}-1$ & $\mathrm{~T}-1$ & $\mathrm{~T}-1$ \\
\cline { 2 - 6 } & $\mathrm{T}-2$ & $\mathrm{~T}-1$ & $\mathrm{~T}-2$ & $\mathrm{~T}-1$ & $\mathrm{~T}-2$ \\
\cline { 2 - 6 } & $\mathrm{T}-3$ & $\mathrm{~T}-1$ & $\mathrm{~T}-1$ & $\mathrm{~T}-3$ & $\mathrm{~T}-3$ \\
\cline { 2 - 6 } & $\mathrm{T}-4$ & $\mathrm{~T}-1$ & $\mathrm{~T}-2$ & $\mathrm{~T}-3$ & $\mathrm{~T}-4$ \\
\hline
\end{tabular}




\subsubsection{Example to prove entry $(1,1)$}

Let $U=\left\{a_{1}, a_{2}, a_{3}, a_{4}, a_{5}, a_{6}, a_{7}, a_{8}\right\}$.

$U / \operatorname{SIM}(P)=\left\{\left\{a_{1}, a_{7}\right\},\left\{a_{2}, a_{3}, a_{4}, a_{5}, a_{6}, a_{7}\right\},\left\{a_{8}\right\}\right\}$

$U / \operatorname{SIM}(Q)=\left\{\left\{a_{1}, a_{7}\right\},\left\{a_{1}, a_{2}, a_{3}, a_{4}, a_{5}, a_{6}, a_{7}, a_{8}\right\}\right.$,

$$
\left.\left\{a_{3}, a_{4}, a_{5}, a_{6}\right\},\left\{a_{8}\right\}\right\}
$$

Let $X=\left\{a_{1}, a_{3}, a_{4}, a_{7}\right\}$.

$\underline{P} X=\left\{a_{1}, a_{7}\right\} \neq \phi$ and $\bar{P} X=\left\{a_{1}, a_{2}, a_{3}, a_{4}, a_{5}, a_{6}, a_{7}\right\} \neq U$.

Thus $X$ is of Type -1 w.r.t. $P$.

$\underline{Q X}=\left\{a_{1}, a_{7}\right\} \neq \phi$ and $\bar{Q} X=\left\{a_{1}, a_{2}, a_{3}, a_{4}, a_{5}, a_{6}, a_{7}\right\} \neq U$.

Thus $X$ is of Type -1 w.r.t. $Q$.

$(P+Q)^{P} X=\left\{a_{1}, a_{7}\right\} \neq \phi$ and

$(\overline{P+Q})^{P} X=\left\{a_{1}, a_{2}, a_{3}, a_{4}, a_{5}, a_{6}, a_{7}\right\} \neq U$.

Thus $X$ is of Type -1 w.r.t. $(P+Q)^{P}$.

\subsubsection{Example to prove entry $(1,3)$}

Let $U=\left\{a_{1}, a_{2}, a_{3}, a_{4}, a_{5}, a_{6}, a_{7}, a_{8}\right\}$.

$U / \operatorname{SIM}(P)=\left\{\left\{a_{1}, a_{7}\right\},\left\{a_{2}, a_{3}, a_{4}, a_{5}, a_{6}, a_{7}\right\},\left\{a_{8}\right\}\right\}$

$U / \operatorname{SIM}(Q)=\left\{\left\{a_{1}, a_{7}\right\},\left\{a_{1}, a_{2}, a_{3}, a_{4}, a_{5}, a_{6}, a_{7}, a_{8}\right\}\right.$, $\left.\left\{a_{3}, a_{4}, a_{5}, a_{6}\right\},\left\{a_{8}\right\}\right\}$

Let $X=\left\{a_{1}, a_{7}, a_{8}\right\}$.

$\underline{P} X=\left\{a_{1}, a_{7}, a_{8}\right\} \neq \phi$ and $\bar{P} X=\left\{a_{1}, a_{7}, a_{8}\right\} \neq U$.

Thus $X$ is of Type -1 w.r.t. P.

$\underline{Q X}=\left\{a_{1}, a_{7}, a_{8}\right\} \neq \phi$ and $\bar{Q} X=U$.

Thus $X$ is of Type -3 w.r.t. $Q$.

$(P+Q)^{P}(X)=\left\{a_{1}, a_{7}, a_{8}\right\} \neq \phi$ and

$(\overline{P+Q})^{P} X=\left\{a_{1}, a_{7}, a_{8}\right\} \neq U$.

Thus $X$ is of Type -1 w.r.t. $(P+Q)^{p}$

\subsection{Table for type of $X^{C}$ with respect to $(P+Q)^{P}$}

This subsection provides the types of complement of pessimistic multi granulation rough set in the following table. Proofs with examples for some of the entries in the table are then given.

\begin{tabular}{|c|c|}
\hline $\mathbf{X}$ & ${ }^{C}$ \\
\hline $\mathrm{T}-1$ & $\mathrm{~T}-1$ \\
\hline $\mathrm{T}-2$ & $\mathrm{~T}-3$ \\
\hline $\mathrm{T}-3$ & $\mathrm{~T}-2$ \\
\hline $\mathrm{T}-4$ & $\mathrm{~T}-4$ \\
\hline
\end{tabular}

\subsubsection{Example to prove entry of row2}

Let $U=\left\{a_{1}, a_{2}, a_{3}, a_{4}, a_{5}, a_{6}, a_{7}, a_{8}\right\}$.

$U / \operatorname{SIM}(P)=\left\{\left\{a_{1}, a_{7}\right\},\left\{a_{2}, a_{3}, a_{4}, a_{5}, a_{6}, a_{7}\right\},\left\{a_{8}\right\}\right\}$

Let $X=\left\{a_{2}, a_{3}, a_{4}\right\}$.

$\underline{P} X=\phi$ and $\bar{P} X=\left\{a_{2}, a_{3}, a_{4}, a_{5}, a_{6}\right\} \neq U$.

Thus $X$ is of Type -2 w.r.t. P.

$X^{C}=\left\{a_{1}, a_{5}, a_{6}, a_{7}, a_{8}\right\}$.

$\underline{P} X^{C}=\left\{a_{1}, a_{7}, a_{8}\right\} \neq \phi$ and

$\bar{P} X^{C}=\left\{a_{1}, a_{2}, a_{3}, a_{4}, a_{5}, a_{6}, a_{7}, a_{8}\right\}=U$.

Thus $X^{c}$ is of Type -3 w.r.t.P

3.3 Table for type of $X \cup Y$ with respect to $(P+Q)^{P}$

This subsection provides the types of union of two pessimistic multi granular rough sets with respect to $\mathrm{P}+\mathrm{Q}$ in the following table. Proofs with examples for some of the entries in the table are then given.

\section{Proof of entry $(1,1)$}

Suppose $\mathrm{X}$ and $\mathrm{Y}$ are both of Type-1. Then

$(P+Q)^{P} X \neq \phi,(P+Q)^{P} Y \neq \phi$,

$\overline{(P+Q)}^{P} X \neq U$ and $\overline{(P+Q)}^{P} Y \neq U$.

From (2.12) it follows that

$\underline{(P+Q)^{P}}(X \cup Y) \neq \phi$.

But using (2.11) we see that

$\overline{(P+Q)}^{P}(X \cup Y)$ has both the possibilities

of being equal or not equal to $U$.

So, $X \cup Y$ can be of Type-1 or of Type -3 .

\begin{tabular}{|c|c|c|c|c|c|}
\hline U & \multicolumn{5}{|c|}{ Type of $\mathbf{Y}$ with respect to $(P+Q)^{P}$} \\
\hline \multirow{4}{*}{$\begin{array}{l}\text { Type of } \\
\mathbf{X} \text { with } \\
\text { respect } \\
\text { to } \\
(P+Q)^{P}\end{array}$} & & T-1 & T-2 & T-3 & T-4 \\
\hline & T-1 & $\begin{array}{l}\text { T-1/ } \\
\text { T-3 }\end{array}$ & T-1/T-3 & T-3 & T-3 \\
\hline & T-2 & $\begin{array}{l}\text { T-1/ } \\
\text { T-3 }\end{array}$ & $\begin{array}{l}\text { T-1/T-2/ } \\
\text { T-3/T-4 } \\
\end{array}$ & T-3 & $\begin{array}{l}\mathrm{T}-3 / \\
\mathrm{T}-4 \\
\end{array}$ \\
\hline & T-3 & T-3 & T-3 & T-3 & T-3 \\
\hline & T-4 & T-3 & T-3/T-4 & T-3 & $\begin{array}{c}\text { T-3/ } \\
\text { T-4 }\end{array}$ \\
\hline
\end{tabular}




\subsubsection{Examples to prove entry $(1,1)$}

Let $U=\left\{a_{1}, a_{2}, a_{3}, a_{4}, a_{5}, a_{6}, a_{7}, a_{8}\right\}$.

$U / \operatorname{SIM}(P)=\left\{\left\{a_{1}, a_{7}\right\},\left\{a_{2}, a_{3}, a_{4}, a_{5}, a_{6}, a_{7}\right\}\right.$, $\left.\left\{a_{8}\right\}\right\}$

$U / \operatorname{SIM}(Q)=\left\{\left\{a_{1}, a_{7}\right\},\left\{a_{1}, a_{2}, a_{3}, a_{4}, a_{5}, a_{6}, a_{7}, a_{8}\right\}\right.$, $\left.\left\{a_{3}, a_{4}, a_{5}, a_{6}\right\},\left\{a_{8}\right\}\right\}$

\section{Case 1}

Let $X=\left\{a_{1}, a_{7}\right\}$ and $Y=\left\{a_{8}\right\}$. Then $X U Y=\left\{a_{1}, a_{7}, a_{8}\right\}$. $(\underline{P+Q})^{P} X=\left\{a_{1}, a_{7}\right\} \neq \phi$ and $(\overline{P+Q})^{p} X=\left\{a_{1}, a_{7}\right\} \neq U$.

Thus $X$ is of Type -1

$(\underline{P+Q})^{P} Y=\left\{a_{8}\right\} \neq \phi$ and $(\overline{P+Q})^{p} Y=\left\{a_{8}\right\} \neq U$.

Thus $Y$ is of Type -1

$\left(\underline{P+Q}^{P}(X U Y)=\left\{a_{1}, a_{7}, a_{8}\right\} \neq \phi\right.$ and

$(\overline{P+Q})^{p}(X U Y)=\left\{a_{1}, a_{7}, a_{8}\right\} \neq U$.

Thus XUY is of Type -1

\section{Case 2}

Let $X=\left\{a_{1}, a_{3}, a_{4}, a_{7}\right\}$ and $Y=\left\{a_{8}, a_{4}, a_{5}\right\}$.

Then $X U Y=\left\{a_{1}, a_{3}, a_{4}, a_{5}, a_{7}, a_{8}\right\}$.

$(\underline{P+Q})^{P} X=\left\{a_{1}, a_{7}\right\} \neq \phi$ and

$(\overline{P+Q})^{p} X=\left\{a_{1}, a_{7}, a_{2}, a_{3}, a_{4}, a_{5}, a_{6}\right\} \neq U$.

Thus $X$ is of Type -1

$$
\begin{aligned}
& (\underline{P+Q})^{P} Y=\left\{a_{8}\right\} \neq \phi \text { and } \\
& (\overline{P+Q})^{P} Y=\left\{a_{8}, a_{3}, a_{4}, a_{5}, a_{6}\right\} \neq U .
\end{aligned}
$$

Thus $Y$ is of Type -1

$(\underline{P+Q})^{P}(X U Y)=\left\{a_{1}, a_{7}, a_{8}\right\} \neq \phi$ and $(\overline{P+Q})^{p}(X U Y)=\left\{a_{1}, a_{2}, a_{3}, a_{4}, a_{5}, a_{6}, a_{7}, a_{8}\right\}=U$.

Thus XUY is of Type -3

\section{Proof of entry $(1,3)$}

Let both $\mathrm{X}$ and $\mathrm{Y}$ be of Type 1 and Type 3. Then from the properties of type 1 and type 3

$$
\begin{aligned}
& \left.{\left(\overline{P+Q}^{P}\right.}^{P} \mathrm{X}\right) \neq \phi, \quad \underline{(P+Q}^{P}(\mathrm{Y}) \neq \phi, \\
& (\overline{P+Q})^{P}(\mathrm{X}) \neq \mathrm{U} \text { and } \quad(\overline{P+Q})^{P}(\mathrm{Y})=\mathrm{U} .
\end{aligned}
$$

So, using (2.11) and (2.12) we get

$\left(\underline{P+Q}^{P}(\mathrm{XUY}) \neq \phi\right.$ and $(\overline{P+Q})^{P}(\mathrm{XUY})=\mathrm{U}$.
Hence XUY is of type 3 only.

The other cases can be similarly established.

\subsubsection{Example to prove entry $(1,3)$ in XUY table}

Let $U=\left\{a_{1}, a_{2}, a_{3}, a_{4}, a_{5}, a_{6}, a_{7}, a_{8}\right\}$.

$U / \operatorname{SIM}(P)=\left\{\left\{a_{1}, a_{7}\right\},\left\{a_{2}, a_{3}, a_{4}, a_{5}, a_{6}, a_{7}\right\},\left\{a_{8}\right\}\right\}$

$U / \operatorname{SIM}(Q)=\left\{\left\{a_{1}, a_{7}\right\},\left\{a_{1}, a_{2}, a_{3}, a_{4}, a_{5}, a_{6}, a_{7}, a_{8}\right\}\right.$, $\left.\left\{a_{3}, a_{4}, a_{5}, a_{6}\right\},\left\{a_{8}\right\}\right\}$

Let $X=\left\{a_{1}, a_{3}, a_{4}, a_{7}\right\}$ and $Y=\left\{a_{1}, a_{4}, a_{5}, a_{7}, a_{8}\right\}$.

$(\underline{P+Q})^{p} X=\left\{a_{1}, a_{7}\right\} \neq \phi$ and $(\overline{P+Q})^{p} X \neq U$.

Thus $X$ is of Type -1 .

$(\underline{P+Q})^{P} Y=\left\{a_{1}, a_{7}, a_{8}\right\} \neq \phi$ and $(\overline{P+Q})^{p} Y=U$.

Thus $Y$ is of Type-3.

$$
\begin{aligned}
& X U Y=\left\{a_{1}, a_{3}, a_{4}, a_{5}, a_{7}, a_{8}\right\} . \\
& (\overline{P+Q})^{p}(X U Y)=\left\{a_{1}, a_{7}, a_{8}\right\} \neq \phi \text { and } \\
& (\overline{P+Q})^{p}(X U Y)=U .
\end{aligned}
$$

Thus XUY is of Type-3.

\subsubsection{Examples to prove entry $(2,2)$ in XUY table}

Let us take the following examples and provide proofs for first two cases.

$$
\begin{aligned}
& \text { Let } U=\left\{a_{1}, a_{2}, a_{3}, a_{4}, a_{5}, a_{6}, a_{7}, a_{8}\right\} . \\
& \begin{aligned}
U / \operatorname{SIM}(P)=\{ & \left\{a_{1}, a_{7}\right\},\left\{a_{2}, a_{3}, a_{4}, a_{5}, a_{6}, a_{7}\right\}, \\
& \left.\left\{a_{8}\right\}\right\}
\end{aligned}
\end{aligned}
$$

$$
\begin{aligned}
U / \operatorname{SIM}(Q)=\{ & \left\{a_{1}, a_{7}\right\},\left\{a_{1}, a_{2}, a_{3}, a_{4}, a_{5}, a_{6}, a_{7}, a_{8}\right\}, \\
& \left.\left\{a_{3}, a_{4}, a_{5}, a_{6}\right\},\left\{a_{8}\right\}\right\}
\end{aligned}
$$

\section{Case 1}

Let $X=\left\{a_{1}, a_{3}, a_{4}\right\}$ and $Y=\left\{a_{7}\right\}$.

$(\underline{P+Q})^{P} X=\phi$ and $(\overline{P+Q})^{P} X=\left\{a_{1}, a_{2}, a_{3}, a_{4}, a_{5}, a_{6}, a_{7}\right\} \neq U$.

Thus $X$ is of Type -2 .

$(\underline{P+Q})^{p} Y=\phi$ and $(\overline{P+Q})^{p} Y=\left\{a_{1}, a_{2}, a_{3}, a_{4}, a_{5}, a_{6}, a_{7}\right\} \neq U$.

Thus $Y$ is of Type-2.

$X U Y=\left\{a_{1}, a_{3}, a_{4}, a_{7}\right\}$.

$\underline{(P+Q)})^{P}(X U Y)=\left\{a_{1}, a_{7}\right\} \neq \phi$ and

$(\overline{P+Q})^{p}(X U Y)=\left\{a_{1}, a_{2}, a_{3}, a_{4}, a_{5}, a_{6}\right\} \neq U$.

Thus XUY is of Type-1. 


\section{Case 2}

Let $X=\left\{a_{3}, a_{4}, a_{5}, a_{6}\right\}$ and

$Y=\left\{a_{3}, a_{4}, a_{5}\right\}$.

$(\underline{P+Q})^{P} X=\phi$ and $(\overline{P+Q})^{P} X=\left\{a_{2}, a_{3}, a_{4}, a_{5}, a_{6}\right\} \neq U$.

Thus $X$ is of Type-2.

$(P+Q)^{p} Y=\phi$ and $(\overline{P+Q})^{p} Y=\left\{a_{2}, a_{3}, a_{4}, a_{5}, a_{6}\right\} \neq U$.

Thus $Y$ is of Type-2.

$X U Y=\left\{a_{3}, a_{4}, a_{5}, a_{6}\right\}$.

$(\underline{P+Q})^{P}(X U Y)=\phi$ and

$(\overline{P+Q})^{p}(X U Y)=\left\{a_{2}, a_{3}, a_{4}, a_{5}, a_{6}\right\} \neq U$

Thus XUY is of Type-2

Then let us take the following examples and provide proofs for the next two cases.

Let $U=\left\{a_{1}, a_{2}, a_{3}, a_{4}, a_{5}, a_{6}, a_{7}, a_{8}\right\}$

$U / \operatorname{SIM}(P)=\left\{\left\{a_{1}, a_{7}\right\},\left\{a_{2}, a_{3}, a_{4}, a_{5}\right\},\left\{a_{6}, a_{8}\right\}\right\}$

$U / \operatorname{SIM}(Q)=\left\{\left\{a_{1}, a_{7}\right\},\left\{a_{1}, a_{2}, a_{3}, a_{4}, a_{5}, a_{6}, a_{7}, a_{8}\right\}\right.$,

$$
\left.\left\{a_{4}, a_{5}, a_{6}\right\},\left\{a_{6}, a_{8}\right\}\right\}
$$

\section{Case 3}

Let $X=\left\{a_{3}, a_{4}, a_{7}\right\}$ and $Y=\left\{a_{1}, a_{8}\right\}$.

$(\underline{P+Q})^{P} X=\phi$ and $(\overline{P+Q})^{P} X=\left\{a_{2}, a_{3}, a_{4}, a_{5}\right\} \neq U$.

Thus $X$ is of Type-2.

$(P+Q)^{P} Y=\phi$ and $(\overline{P+Q})^{P} Y=\left\{a_{1}, a_{2}, a_{3}, a_{7}, a_{8}\right\} \neq U$.

Thus $Y$ is of Type-2.

$X U Y=\left\{a_{1}, a_{3}, a_{4}, a_{6}, a_{7}\right\} .(P+Q)^{P}(X U Y)=\left\{a_{1}, a_{7}\right\} \neq \phi$

and $(\overline{P+Q})^{p}(X U Y)=U$.

Thus XUY is of Type-3.

\section{Case 4}

Let $X=\left\{a_{3}, a_{4}, a_{7}\right\}$ and $Y=\left\{a_{6}, a_{2}\right\}$.

$(P+Q)^{P} X=\phi$ and $(\overline{P+Q})^{P} X \neq U$.

Thus $X$ is of Type-2.

$(P+Q)^{P} Y=\phi$ and $(\overline{P+Q})^{P} Y \neq U$.

Thus $Y$ is of Type-2.
$X U Y=\left\{a_{2}, a_{3}, a_{4}, a_{6}, a_{7}\right\}$.

$(P+Q)^{P}(X U Y)=\phi$ and $(\overline{P+Q})^{p}(X U Y)=U$.

Thus XUY is of Type-4

3.4 Table for type of $X \cap Y$ with respect to $(P+Q)^{P}$

This subsection provides the types of intersection of two pessimistic multi granular rough sets with respect to $\mathrm{P}+\mathrm{Q}$ in the following table. Proofs with examples for some of the entries in the table are then given.

\begin{tabular}{|c|c|c|c|c|c|}
\hline$\bigcap$ & \multicolumn{5}{|c|}{ Type of $\mathbf{Y}$ with respect to $(P+Q)^{P}$} \\
\hline \multirow{5}{*}{$\begin{array}{l}\text { Type of } \\
\mathbf{X} \text { with } \\
\text { respect } \\
\quad \text { to } \\
(P+Q)^{P}\end{array}$} & & T-1 & T-2 & T-3 & T-4 \\
\hline & T-1 & T-1/T-2 & T-2 & T-1/T-2 & T-2 \\
\hline & T-2 & T-2 & T-2 & T-2 & T-2 \\
\hline & T-3 & T-1/T-2 & T-2 & $\begin{array}{l}\text { T-1/T-2/ } \\
\text { T-3/T-4 }\end{array}$ & $\begin{array}{l}\text { T-2 } \\
/ \mathrm{T}-4\end{array}$ \\
\hline & T-4 & T-2 & T-2 & T-2/T-4 & $\begin{array}{c}\text { T-2 } \\
/ \mathrm{T}-4\end{array}$ \\
\hline
\end{tabular}

\section{Proof of entry $(1,3)$}

Suppose $\mathrm{X}$ is of Type- 1 and $\mathrm{Y}$ is of Type- 3 . Then

$(P+Q)^{P} X \neq \phi,(P+Q)^{P} Y \neq \phi$,

$\overline{(P+Q)}^{P} X \neq U$ and $\overline{(P+Q)}^{P} Y=U$.

From (2.13) it follows that

$\overline{(P+Q)}^{P}(X \cap Y) \neq U$.

But using (2.10) we see that

$(P+Q)^{P}(X \cap Y)$ has both the possibilities

of being or not being equal to $\phi$.

So $X \cap Y$ can be of Type-1orType -2

\subsubsection{Examples to prove entry $(1,3)$}

Let $U=\left\{a_{1}, a_{2}, a_{3}, a_{4}, a_{5}, a_{6}, a_{7}, a_{8}\right\}$.

$U / \operatorname{SIM}(P)=\left\{\left\{a_{1}, a_{7}\right\},\left\{a_{2}, a_{3}, a_{4}, a_{5}, a_{6}, a_{7}\right\}\right.$,

$\left.\left\{a_{8}\right\}\right\}$

$U / \operatorname{SIM}(Q)=\left\{\left\{a_{1}, a_{7}\right\},\left\{a_{1}, a_{2}, a_{3}, a_{4}, a_{5}, a_{6}, a_{7}, a_{8}\right\}\right.$, $\left.\left\{a_{3}, a_{4}, a_{5}, a_{6}\right\},\left\{a_{8}\right\}\right\}$

\section{Case 1}

Let $X=\left\{a_{2}, a_{3}, a_{4}, a_{5}, a_{6}\right\}$ and $Y=\left\{a_{2}, a_{3}, a_{4}, a_{5}, a_{6}, a_{7}, a_{8}\right\}$.

Then $X \cap Y=\left\{a_{2}, a_{3}, a_{4}, a_{5}, a_{6}\right\}$. 
$(\underline{P+Q})^{P} X \neq \phi$ and $(\overline{P+Q})^{P} X=\left\{a_{2}, a_{3}, a_{4}, a_{5}, a_{6}\right\} \neq U$.

Thus $X$ is of Type -1 .

$(\underline{P+Q})^{p} Y \neq \phi$ and $(\overline{P+Q})^{P} Y=\left\{a_{1}, a_{2}, a_{3}, a_{4}, a_{5}, a_{6}, a_{7}, a_{8}\right\}=U$.

Thus $Y$ is of Type -3 .

$(\underline{P+Q})^{P}(X \cap Y) \neq \phi$ and $(\overline{P+Q})^{P}(X \cap Y) \neq U$.

Thus $X \cap Y$ is of Type-1.

\section{Case 2}

Let $X=\left\{a_{3}, a_{4}, a_{5}, a_{8}\right\}$ and $Y=\left\{a_{1}, a_{2}, a_{3}, a_{4}, a_{5}, a_{6}, a_{8}\right\}$.

Then $X \cap Y=\left\{a_{3}, a_{4}, a_{5}\right\}$.

$(\underline{P+Q})^{P} X=\left\{a_{8}\right\} \neq \phi$ and $(\overline{P+Q})^{P} X=\left\{a_{2}, a_{3}, a_{4}, a_{5}, a_{6}, a_{8}\right\} \neq U$.

Thus $X$ is of Type -1 .

$\left(\underline{P+Q}^{p} Y=\left\{a_{8}\right\} \neq \phi\right.$ and $(\overline{P+Q})^{P} Y=\left\{a_{1}, a_{2}, a_{3}, a_{4}, a_{5}, a_{6}, a_{7}, a_{8}\right\}=U$.

Thus $Y$ is of Type -3 .

$(\underline{P+Q})^{P}(X \cap Y)=\phi$ and $(\overline{P+Q})^{P}(X \cap Y) \neq U$.

Thus $X \cap Y$ is of Type-2.
Let $\mathrm{X}$ and $\mathrm{Y}$ be of Type 2 and Type 1 respectively. Then from the properties of type 2 and type 1 multi granular rough sets we get $\underline{(P+Q)}^{P}(\mathrm{X})=\phi$, $\underline{(P+Q)}^{P}(\mathrm{Y})=\phi,\left(\overline{P+Q}^{P}(\mathrm{X}) \neq \mathrm{U}\right.$ and $(\overline{P+Q})^{P}(\mathrm{Y})$ $\neq \mathrm{U}$.

So using properties (2.10) and (2.13) we get $\underline{(P+Q)}^{P}(\mathrm{X} \cap \mathrm{Y})=\phi$ and $(\overline{P+Q})^{P}(\mathrm{X} \cap \mathrm{Y}) \neq \mathrm{U}$. So, $X \cap Y$ is of type 2 . This completes the proof. The other cases can be established similarly.

\subsubsection{Example to prove entry $(2,1)$}

Let $U=\left\{a_{1}, a_{2}, a_{3}, a_{4}, a_{5}, a_{6}, a_{7}, a_{8}\right\}$.

$U / \operatorname{SIM}(P)=\left\{\left\{a_{1}, a_{7}\right\},\left\{a_{2}, a_{3}, a_{4}, a_{5}, a_{6}, a_{7}\right\}\right.$,

$$
\left.\left\{a_{8}\right\}\right\}
$$

$U / \operatorname{SIM}(Q)=\left\{\left\{a_{1}, a_{7}\right\},\left\{a_{1}, a_{2}, a_{3}, a_{4}, a_{5}, a_{6}, a_{7}, a_{8}\right\}\right.$, $\left.\left\{a_{3}, a_{4}, a_{5}, a_{6}\right\},\left\{a_{8}\right\}\right\}$

\section{Proof of entry $(2,1)$}

Let $X=\left\{a_{3}, a_{4}, a_{5}, a_{8}\right\}$ and $Y=\left\{a_{3}, a_{4}, a_{5}\right\}$.

Then $X \cap Y=\left\{a_{3}, a_{4}, a_{5}\right\}$.

$(\underline{P+Q})^{P} X=\left\{a_{8}\right\} \neq \phi$ and $(\overline{P+Q})^{P} X=\left\{a_{2}, a_{3}, a_{4}, a_{5}, a_{6}, a_{8}\right\} \neq U$.

Thus $X$ is of Type -1 .

$(\underline{P+Q})^{p} Y=\phi$ and $(\overline{P+Q})^{P} Y=\left\{a_{2}, a_{3}, a_{4}, a_{5}, a_{6}\right\} \neq U$.

Thus $Y$ is of Type -2 .

$\left(\underline{P+Q}^{P}(X \cap Y)=\phi\right.$ and $(\overline{P+Q})^{P}(X \cap Y)=\left\{a_{2}, a_{3}, a_{4}, a_{5}, a_{6}\right\} \neq U$.

\section{Thus $X \cap Y$ is of Type-2}

\section{Conclusion}

Two types of multi-granular rough sets have been introduced in the literature ([10], [13]). Topological properties of optimistic multigranular rough sets were studied by Tripathy et al ([17]). In this paper we studied the topological properties of pessimistic multi granular rough sets with respect to the three set theoretic operations of union, intersection and complementation. The tables show that there are multiple answers to some of the cases as like as the case of basic rough sets. These multiple answers specify impreciseness and ambiguity in information that is available with the user to classify object of a universe. Thus the models discussed in this paper are rightly suitable for handling impreciseness in data in more effective and elegant manner using both types of multi-granular rough sets. Also, we provided examples in some cases to illustrate the fact that the multiple answers can actually occur. These results can be used in approximation of classifications and rule induction. Also our results hold true for both complete and incomplete pessimistic multigranulation systems

\section{References}

[1] Kryszkiewicz, K.: Rough set approach to incomplete information systems, Information Sciences, vol.112, (1998), pp.39- 49. 
[2] Liang, J.Y and Shi, Z.Z.: The information entropy, rough entropy and knowledge granulation in rough set theory, International Journal of Uncertainty, Fuzziness and Knowledge-Based Systems, vol.12(1),(2001),pp. $37-46$.

[3] Liang, J.Y, Shi, Z.Z., Li, D. Y. and Wierman, M. $\mathrm{J} .:$ The information entropy, rough entropy and knowledge granulation in incomplete information system, International Journal of general systems, vol.35(6), (2006),pp.641 - 654.

[4] Liang, J.Y and Li, D. Y.: Uncertainty and Knowledge acquisition in Information Systems, Science Press, Beijing, China, (2005).

[5] Pawlak, Z., Rough sets, Int. jour. of Computer and Information Sciences,11, (1982), pp.341-356.

[6] Pawlak, Z.: Rough sets: Theoretical aspects of reasoning about data, Kluwer academic publishers (London), (1991).

[7] Pawlak, Z. and Skowron, A., Rudiments of rough sets, Information Sciences-An International Journal, Elsevier Publications, 177(1), (2007), pp.3-27.

[8] Pawlak, Z. and Skowron, A., Rough sets: Some extensions, Information Sciences-An International Journal, Elsevier Publications, 177(1), (2007), pp.28-40.

[9] Pawlak, Z. and Skowron, A., Rough sets and Boolean reasoning,. Information Sciences-An International Journal, Elsevier Publications, 177(1), (2007), pp. 41-73.

[10] Qian, Y.H and Liang, J.Y.: Rough set method based on Multi-granulations, Proceedings of the $5^{\text {th }}$ IEEE Conference on Cognitive Informatics, vol.1, (2006),pp.297 - 304.

[11] Qian, Y.H, Liang, J.Y. and Dang, C.Y.: MGRS in Incomplete Information Systems, IEEE Conference on Granular Computing,(2007),pp.163 -168 .

[12] Qian, Y.H, Liang, J.Y. and Dang, C.Y.: Incomplete Multigranulation Rough set, IEEE Transactions on Systems, Man and Cybernetics Part A: Systems and Humans, Vol.40, No.2, March 2010, pp.420 - 431.

[13] Qian, Y.H., Liang, J.Y and Dang, C.Y.: Pessimistic rough decision, proceedings of RST 2010, Zhoushan, China, (2010), pp. 440-449.

[14] Tripathy, B.K.: On Approximation of classifications, rough equalities and rough equivalences, Studies in Computational Intelligence, vol.174, Rough Set Theory: A True Landmark in Data Analysis, Springer Verlag, (2009), pp.85 - 136.

[15] Tripathy, B.K.: Rough Sets on Fuzzy Approximation Spaces and Intuitionistic Fuzzy Approximation Spaces, Studies in Computational Intelligence, vol.174, Rough Set Theory: A True
Landmark in Data Analysis, Springer Verlag, (2009), pp.03 - 44.

[16] Tripathy, B.K. and Mitra, A.: Topological Properties of Rough Sets and their Applications, International Journal of Granular Computing, Rough Sets and Intelligent Systems (IJGCRSIS), (Switzerland),vol.1, no.4, (2010),pp.355-369.

[17] Tripathy, B.K. and Raghavan, R.: On Some Topological Properties of Multigranular Rough Sets, Journal of Advances in Applied science Research, Vol.2, no.3, (2011), pp.536-543.

Dr. B.K Tripathy is a senior professor in the school of computing sciences and engineering, VIT University, at Vellore, India, has published more than 150 technical papers in international journals/ proceedings of international conferences/ edited book chapters of reputed publications like Springer and guided 12 students for $\mathrm{PhD}$. so far. $\mathrm{He}$ is having more than 30 years of teaching experience. $\mathrm{He}$ is a member of international profes sional as sociations like IEEE, ACM, IRSS, CSI, IMS, OITS, OMS, IACSIT, IST, ACEEE and is a reviewer of around 20 international journals which include IEEE, World Scientific, Springer and Science Direct publications. Also, he is in the editorial board of at least 10 international journals. His current research interest includes Fuzzy sets and systems, Rough sets and knowledge engineering, Granular computing, soft computing, bag theory, data clustering, database anonymisation, list theory and social network analys is.

M. Nagaraju is a assistant professor (senior) in the school of computing sciences and engineering, VIT University, Vellore, India. He is pursuing his Ph.D. Degree in computer science in the same school. He is a life member in professional associations like ISTE and CSI. His current research interest includes Fuzzy sets and systems, Rough sets and knowledge engineering, Granular computing, Database systems, Expert Systems, Data Mining and Decision Support Systems.

How to cite this paper: B.K.Tripathy ,M. Nagaraju,"On Some Topological Properties of Pessimistic Multigranular Rough Sets", International Journal of Intelligent Systems and Applications(IJISA), vol.4, no.8, pp.10-17, 2012. DOI: 10.5815/ijisa.2012.08.02 